\title{
Radiotherapy in COVID-19 patient affected by multiple myeloma: a case report
}

\author{
Marco Krengli ${ }^{1,2}$, Debora Beldì ${ }^{1}$, Eleonora Ferrara ${ }^{1}$, Micol Zannetti ${ }^{1,2}$, Federico Mastroleo ${ }^{1,2}$, \\ Lorenzo De Paoli ${ }^{3}$, Mariangela Greco ${ }^{3}$, Erica Matino ${ }^{4}$, Mario Pirisi ${ }^{4}$, Gianluca Gaidano ${ }^{3}$ \\ ${ }^{1}$ Division of Radiation Oncology, University Hospital "Maggiore della Carità", Novara, Italy; ${ }^{2}$ Department of Translational Medicine, University \\ of "Piemonte Orientale" Novara, Italy; ${ }^{3}$ Division of Haematology, University Hospital "Maggiore della Carità", Novara, Italy; ${ }^{4}$ Division of Internal \\ Medicine, University Hospital "Maggiore della Carità, Novara, Italy \\ Correspondence to: Marco Krengli, MD. Chair of Radiation Oncology, Department of Translational Medicine, University of "Piemonte Orientale", \\ Via Solaroli, 17 - 28100 Novara, Italy. Email: marco.krengli@med.uniupo.it.
}

\begin{abstract}
In COVID-19 pandemic, cancer patients may be vulnerable for their immunological status and need of immunosuppressive anti-neoplastic treatments. Choosing the best treatment option in COVID-19 positive cancer patients is still a challenging issue. We report the case of a 62-year-old woman diagnosed with multiple myeloma and affected by COVID-19. After the diagnosis of multiple myeloma in January 2019, the patient underwent first line therapy followed by bone marrow autologous stem cell transplantation, achieving a complete response in September 2019. In March 2020, the patient showed intrathoracic progression of the disease, resulting in a severe dysphagia and concomitant positivity to SARS-CoV-2 swab test, cough, fever, and dyspnea related to the involvement of the lung parenchyma as shown by CT-scan. After her admittance to a COVID-19 dedicated inward, she was administered oral hydroxychloroquine and darunavir-cobicistat for 7 days with stabilization of her general clinical conditions. For the worsening of dysphagia, after multidisciplinary discussion, it was decided to deliver radiotherapy to the mediastinal and paravertebral mass with 8 Gy single fraction. After 5 days, her clinical conditions improved, with reduction of dysphagia. The CT confirmed a partial response with reduction of the mass of about $50 \%$. Viral clearance was confirmed by triple negative search for SARS-CoV-2 on nasopharyngeal swabs, one month after first documentation of positivity. Unfortunately, the patient died three months later due to a pulmonary mycotic infection causing respiratory failure. To our knowledge, this case report describes the first experience of mediastinal radiotherapy in a COVID-19 patient affected by myeloma reported in the literature. In case of clinical indication, even in presence of SARS-CoV-2 infection, radiotherapy can be safely delivered and might be considered a treatment option as shown by our experience in this challenging case of intrathoracic myeloma.
\end{abstract}

Keywords: COVID-19; radiotherapy; palliative treatment; multiple myeloma; case report

Submitted May 24, 2020. Accepted for publication Oct 09, 2020.

doi: $10.21037 /$ tcr-20-2172

View this article at: http://dx.doi.org/10.21037/tcr-20-2172

\section{Introduction}

On $11^{\text {th }}$ of March 2020, WHO declared CoVID-19 as pandemic disease, due to its rapid escalation all over the world. Since the beginning of the pandemic, Italy has scored more than 280,000 cases (1), becoming one of the most affected countries.

In most cases, SARS-CoV-2 infections are asymptomatic or present with mild symptoms such as fever, fatigue, myalgia, dry cough, gastrointestinal symptoms (nausea, vomit, and diarrhea) (2). In severe cases, dyspnea and respiratory failure occurs. These symptoms are generally associated with a typical computed tomography (CT) scan pattern that shows an interstitial pneumonia, with an interlobular septal thickening appearing in a crazypaving and/or ground glass opacity, suggestive for a viral 
respiratory infection (3).

In this scenario, cancer patients may be more vulnerable due to their immunological status and the need of immunosuppressive therapies such as anti-neoplastic treatments (4). In daily practice, making appropriate treatment decision requires a careful balance of risks and benefits. The risk-benefit ratio during a pandemic should suit to each patient's need, focusing on the goal of treatment: for instance, palliative treatments should be carefully considered depending on the performance status of the patient, the severity of symptoms and the absence of alternative treatments (5).

By collecting the experience on an emerging number of radiation oncology departments facing COVID-19 $(6,7)$, different analysis on the risk-benefit ratio of treatments have been recently performed (8-10), trying to define a consensus.

Although literature data are still scarce to draw conclusion, what emerges from studies investigating the impact of SARS-CoV-2 infection on cancer patients is the higher mortality rate and the risk of developing severe complications related to COVID-19. Moreover, in most cases reported in the literature, treatments, including radiotherapy, were delayed until SARS-CoV-2 negativization, in order to minimize adverse events and risk of in-hospital spread among patients and health workers $(11,12)$.

The novelty of our case report is related to the decision to perform an elective radiation treatment in a SARS$\mathrm{CoV}-2$ positive patient, symptomatic for dysphagia related to a mediastinal paravertebral localization of multiple myeloma (MM) determining esophageal compression. The cornerstone of the treatment for MM is systemic therapy with anti-myeloma drugs; radiotherapy (RT) plays an important supportive role especially in relieving symptoms derived from tumor masses (i.e., plasmacytomas) in bone or soft tissue (13).

We describe our experience including the decisionmaking process, the adopted protective measures, as well as the early outcome after palliative radiotherapy.

We present the following case in accordance with the CARE reporting checklist (available at http://dx.doi. org/10.21037/tcr-20-2172).

\section{Case presentation}

We report the case of a 62-year-old woman, diagnosed with multiple myeloma at the beginning of 2019. She had a previous history of silent monoclonal gammopathy (MGUS) diagnosed in 1994, hypercholesterolemia and osteoporosis. She was surgically treated at first with ovary resection in 1981 and then with hysteroannessiectomy in 2000 for uterine fibromatosis.

She also experienced a previous event of viral interstitial pneumonia from H1N1 in 2019, which required admission to intensive care unit and treatment with oseltamivir and antibiotics.

At the end of 2018, she performed magnetic resonance imaging (MRI) and bone scintigraphy due to stubbing lower back pain, showing multiple lesions in thoraco-lumbarsacral vertebra, costal bones, and hip bone. The patient was addressed to hematological consultation on January 2019 to investigate a possible plasma cell neoplasia. Bone marrow biopsy revealed a plasma cellular infiltration of $65 \%$ and FISH analysis did not reveal cytogenetic abnormalities among the ones that were tested. Blood tests showed a serum monoclonal component (sMC) IgG/lambda $1.3 \mathrm{~g} / \mathrm{dL}$ and an abnormal FLC ratio of 0.37 (K: $8 \mathrm{mg} / \mathrm{L}$; L: $22 \mathrm{mg} / \mathrm{L}$ ); BenceJones proteinuria was absent and there was no anemia, hypercalcemia or renal failure. Albumin and Beta-2microglobulinemia showed normal value, while LDH level was 2-fold upper normal limit value. A diagnosis of multiple myeloma stage I according to international staging system (ISS) and stage IIIA according to Salmon Durie staging system was established.

The patient started induction of 4 VTD cycles (bortezomib-thalidomide-dexamethasone) from January to May 2019, achieving a complete response according to biochemical restaging and imaging by $\mathrm{CT}$ and positron emission tomography (PET)-CT. In June 2019, hematopoietic stem cell mobilization treatment with intermediate dose of cyclophosphamide $\left(2 \mathrm{~g} / \mathrm{m}^{2}\right)$ was performed obtaining an adequate harvest of peripheral blood stem cells (PBSC). Subsequently, in September 2019, the patient underwent high-dose conditioning chemotherapy with melphalan $200 \mathrm{mg} / \mathrm{m}^{2}$ followed by autologous stem cell transplantation, achieving a complete response.

On $5^{\text {th }}$ March 2020, the patient was admitted to the emergency room of the University Hospital "Maggiore della Carità", Novara, Piedmont, Italy. She presented with persistent fever ( $38^{\circ}$ temperature for 10 days), despite antibiotic therapy, and dysphagia. She was in fair general conditions (ECOG performance status 1), blood tests revealed high CRP level $(11.79 \mathrm{mg} / \mathrm{dL})$, moderatelow arterial $\mathrm{O}_{2}$ pression $(72 \mathrm{mmHg})$ and arterial $\mathrm{O}_{2}$ 



Figure 1 High-resolution Computed Tomography without contrast performed on 19th March at the time of COVID-19 diagnosis, showing: (A) bilateral pleural and pericardial effusion with ground glass areas in the right parenchyma and (B) mediastinal mass extending into the left paravertebral region (yellow arrow) displacing the esophagus (pink arrow). High-resolution Computed Tomography with contrast performed on 29th April, showing: (C) improvement of pleural effusion without worsening of the ground glass areas and (D) partial response to radiotherapy with reduction of pathological mediastinal tissue (yellow arrow) without esophageal displacement (pink arrow).

saturation of $95 \%$. She was tested for H1N1 and other respiratory flu viruses, chlamydia, mycoplasma, legionella, cytomegalovirus, campylobacter, aspergillus. While waiting for the results of blood and urine cultural tests (which were all negative), she was admitted to the Internal Medicine inward. The patient was awake and collaborative; $\mathrm{O}_{2}$ saturation in open air was $98 \%$. Medical examination revealed crackles at left lung base, with a reduction of vesicular murmur.

On $16^{\text {th }}$ March, the patient showed the biochemical progression of the disease: $\mathrm{Hb}$ was $9.3 \mathrm{~g} / \mathrm{dL}$, sMC was present $(1,400 \mathrm{mg} / \mathrm{dL})$, while Bence-Jones proteinuria, hypercalcemia and renal insufficiency were absent.

On $18^{\text {th }}$ March, the nasopharyngeal swab tested positive for COVID-19: the patient was shifted to a COVID-19 dedicated inward. High-resolution CT (HRCT), performed on $19^{\text {th }}$ March, showed ground-glass areas in subpleural pulmonary parenchyma of the lingula and in the anterior segment of the right upper lobe. There was bilateral pleural effusion, especially on the left side, with a measured depth of $4.2 \mathrm{~cm}$. Concomitant pericardial effusion was reported, with a measured depth of $2 \mathrm{~cm}$. CT also revealed paravertebral pathological tissue, which extended from D7 to D12 for about $11.5 \mathrm{~cm}$ cranio-caudal and $9 \mathrm{~cm} \times 6 \mathrm{~cm}$ in axial plan. It caused anterior deviation of the heart and compression of the esophagus and the aorta (Figure 1). Biochemical restaging also showed a reappearance of sMC. These data demonstrated a very early and aggressive relapse of the disease.

As therapy directed toward SARS-CoV-2 infection, the patient was administered oral hydroxychloroquine at a dose of $200 \mathrm{mg}$ bid for 14 days and darunavir-cobicistat at a dose of $800 / 150 \mathrm{mg}$ daily for 7 days with stabilization of her general clinical conditions, resulting in an amelioration of cough and a lowering of body temperature. Moreover, corticosteroid therapy was started for the persistence of dysphagia: no symptomatic improvement was achieved. Therefore, the case was discussed in a multidisciplinary meeting among Hematologists, Internists and Radiation Oncologists, focusing the attention on the elevated risk of infection, comorbidity, and death in case of systemic myeloma treatment (with proteasome inhibitors, 


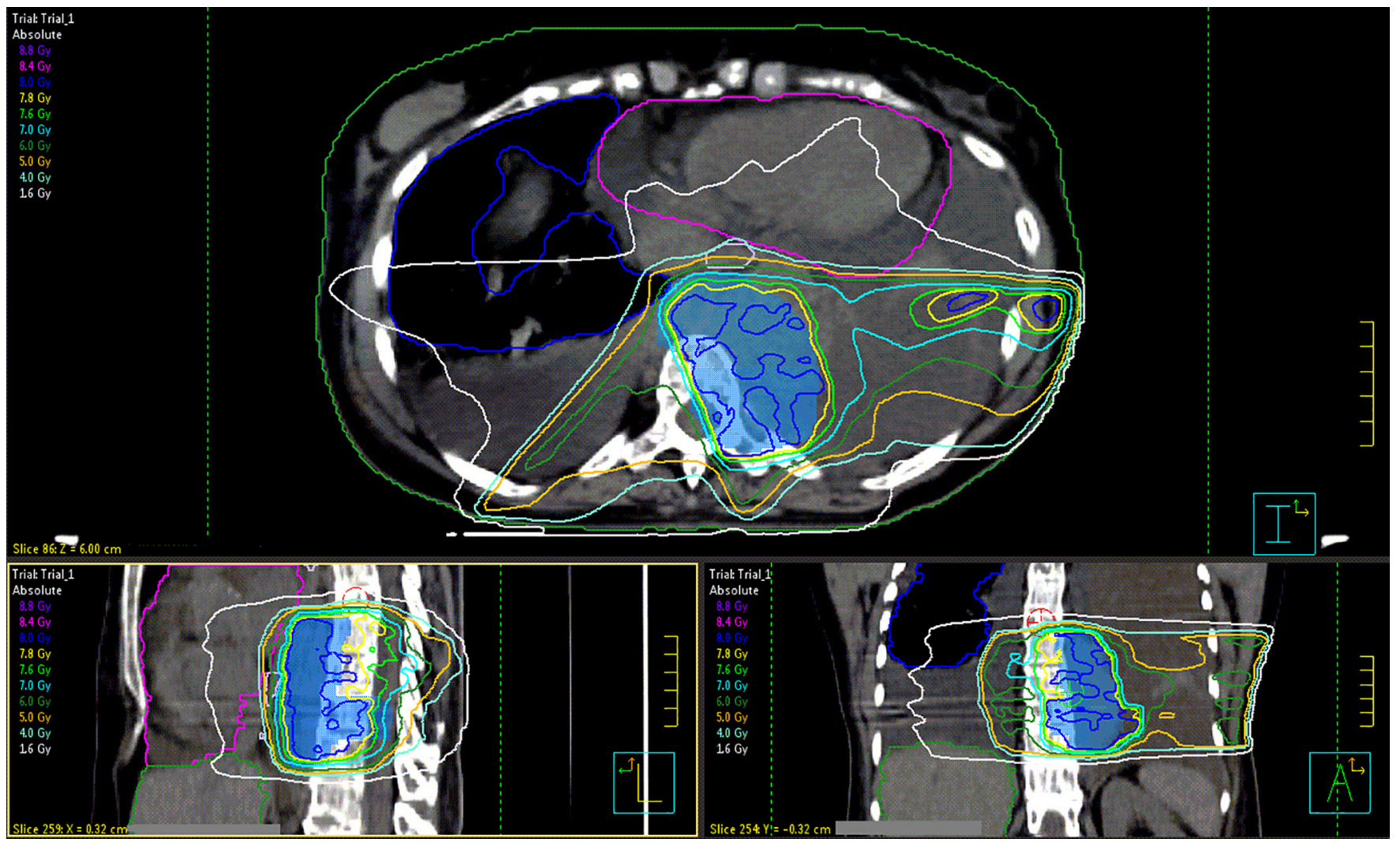

Figure 2 Treatment plan in axial, sagittal and coronal views with $6 \mathrm{MV}$ X-rays by intensity modulated radiotherapy to 8 Gy in single fraction.

monoclonal antibody or immunomodulatory drugs) during a moderate-severe SARS-CoV-2 infection. For these reasons, it was decided to consider radiation therapy to the mediastinal mass, finalized to palliate dysphagia and obtain a bridge towards a systemic treatment at the recovery of COVID-19.

On $31^{\text {st }}$ March, CT planning was performed: the patient was under $\mathrm{O}_{2}$ support with nasal cannula without severe dyspnea. Body temperature was $37.4^{\circ}$, no neurological deficits or pain were reported.

Simulation-CT confirmed the presence of the mediastinal lesion extending in the left paravertebral space and revealed the worsening of pulmonary conditions with an increase of the bilateral pleural effusion. The patient underwent thoracentesis on the left side and cytologic examination resulted in a sample rich in plasma cells $(>70 \%)$ belonging to the MM clone.

After a new multidisciplinary evaluation, a symptomatic and cytoreductive radiotherapy approach in single administration on the mediastinal and paravertebral bulk was decided, considering the result of the cytologic examination and the persistence of dysphagia, despite SARS-CoV-2 positivity.

On $3^{\text {rd }}$ April, the patient received radiation treatment with IMRT technique, $6 \mathrm{MV} \mathrm{X}$ photons, 8 Gy single fraction on the mediastinal and left paravertebral bulk, trying to spare the lung parenchyma and the heart volume as much as possible (Figure 2). Prophylactic febuxostat $80 \mathrm{mg}$ was administered, by monitoring of cytolysis signs (i.e., $\mathrm{LDH}, \mathrm{CK}, \mathrm{ALP})$, to avoid renal failure.

Both CT-simulation and treatment delivery were performed respecting protective measures established in agreement with the Medical Direction of our Institution. The patient wore surgical mask during both procedures. Two radiation technologists and a radiation oncologist wore single-use personal protective equipment (PPE): surgicalcap, eye-protection, filtering facepiece respirator FFP2, gown, 2 pairs of gloves and closed shoes. Furthermore, a personal timetable and itinerary were identified. She was scheduled as the last appointment of the day in the 


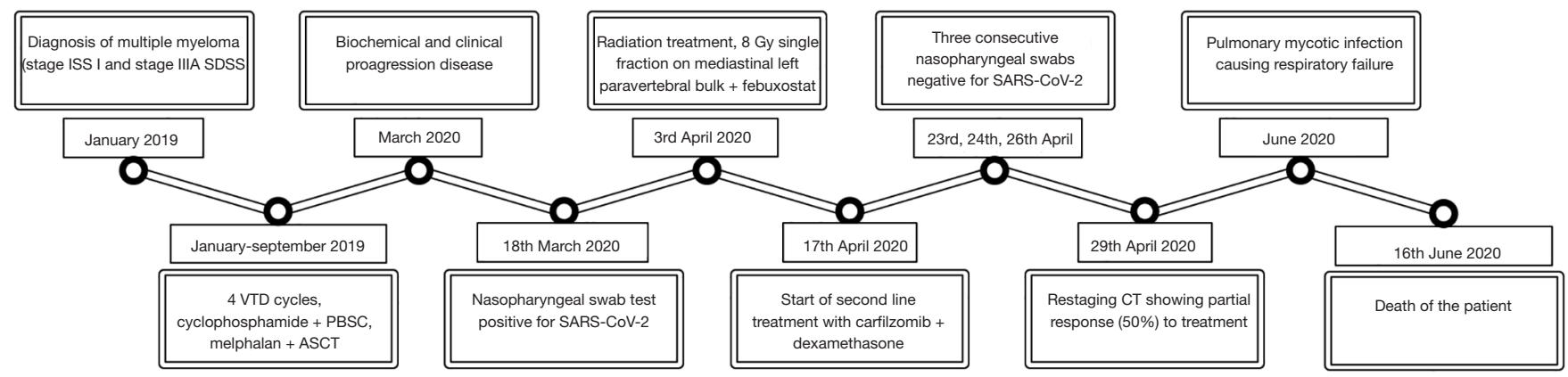

Figure 3 Patient's major events timeline.

timetable of CT planning and treatment delivery, in order to avoid contacts with other patients and to minimize the exposure of healthcare professionals to the only necessary number for treatment. At the end of each procedure, all the machineries, CT room and treatment room were carefully sanitized for the next day.

After 5 days, the patient reported a progressive decreasing of dysphagia symptoms, despite the persistence of SARS-CoV-2 positivity at subsequent nasopharyngeal swab tests.

Due to the fast growth of extra medullary masses related to the hematological disease, on $17^{\text {th }}$ April, the patient started anti-myeloma second-line treatment with carfilzomib $\left(20 \mathrm{mg} / \mathrm{m}^{2}\right)+$ intravenous dexamethasone, 2 days a week for three weeks every four.

On $23^{\text {rd }}, 24^{\text {th }}$ and $26^{\text {th }}$ April respectively, three consecutive SARS-CoV-2 swabs tested negative, so the patient was admitted to Hematology inward.

After the first 6 administrations of carfilzomib and RT, the restaging CT, performed on $29^{\text {th }}$ April, showed a partial response to treatment: the pathological mediastinal tissue extending into the left paravertebral aspect reduced from $9 \mathrm{~cm} \times 6 \mathrm{~cm}$ to $5 \mathrm{~cm} \times 2.5 \mathrm{~cm}$ in axial plan. The pleural effusion flap on the left side was reduced, with a maximum A-P thickness of about $2 \mathrm{~cm}$, versus $4.2 \mathrm{~cm}$, while the small pericardial and the right pleural effusion did not show any change. The ground glass areas, in the subpleural lingual parenchyma and in the anterior segment of the right upper lobe, were substantially unchanged. No radiation-related imaging changes nor clinical adverse events were observed.

At the end of May, patient's conditions started to worsen. She experienced persistent fever and progressive dyspnea, without recurrence of dysphagia. Blood and urine cultural tests and SARS-CoV-2 nasopharyngeal swab test were all negative; the high dosage of beta-D-glucano suggested a mycotic infection. A new CT-scan confirmed this hypothesis showing a typical picture of lung mycosis without evidence of myeloma progression. The patient was first administered isavuconazolo i.v. and then ambisone i.v. unfortunately with no benefit. The patient experienced progressive respiratory failure requiring oxygen therapy. She died on $16^{\text {th }}$ June.

A timeline of patient's major events is available in Figure 3.

All the procedures followed were in accordance with the ethical standards of the responsible committee on human experimentation and with the Helsinki Declaration (as revised in 2013). Written informed consent was obtained from the patient.

\section{Discussion}

In the context of a pandemic, the evaluation of risk benefit ratio of treatments may be a challenge. Oncological treatments must be guaranteed as life-saving treatments, but also palliative treatments require a critical analysis of risk, expected benefits and alternatives (14). In absence of level I evidence, it is currently difficult to estimate the consequences of COVID-19 for cancer patients and physicians should be guided by the best available evidence.

Our case focuses on the risk-benefit balance of a palliative radiation treatment in a relapsed/refractory multiple myeloma patient, considering the expected prognosis, the alternative therapeutic options, and the radiation treatment modalities in terms of high-precision technique and singleshot irradiation.

The severe dysphagia the patient experienced, despite the overall performance status, made the need of palliative treatment not deferrable. Alternative therapeutic options, like systemic anti-MM agents or other palliative medical treatments to allow self-feeding 
of the patient were also considered.

From the multidisciplinary evaluation, there was a consensus about the use of a radiotherapeutic approach as the one with the potentially lowest risk and largest benefit for the patient. As reported by literature data, there is no higher risk of severe events related to SARS-CoV-2 infection for RTpatients compared to non-cancer-patients (10).

In order to reduce exposure to the risk of contagion of healthcare staff and of the other patients, a single session approach was preferred. This therapeutic decision is also supported by recent literature data, which show that even a single fraction RT treatment is effective in symptoms relief (15). Moreover, this therapeutic option has avoided the possibility of treatment interruption due to a worsening of the clinical conditions related to COVID-19. In fact, it is worth to highlight how the stability of patient's respiratory symptoms and clinical parameters contrasted with the worsening of the pulmonary radiological imaging.

Indeed, it is important to highlight that, in radiation oncology departments, treatment equipment is static and used for different patients in constant sequence raising the possibility of cross contagion. So that, a precise protocol of patient management is fundamental to decrease the possibility of contamination. Different measures were taken by health workers in order to prevent infection: during the management of the patient they had to wear FFP2 masks, disposable surgical coats and shoes, surgical caps, gloves, and protective visors. During the procedures, the patient had to wear a surgical mask. All the logistic operations were optimized to lower as much as possible the permanence of the patient in the Radiation Oncology department. Furthermore, the CT and the treatment beds were sterilized by appropriate alcoholic solution before and after they were used by the patient; the same was performed for all the surfaces the patient had been in contact with.

Treatment of COVID-19 positive cancer patients is a challenging issue. The occurrence of COVID-19 pandemic requires a careful risk-benefit analysis in treating or postponing cancer treatments. In COVID-19infected cancer patients scheduled for radiotherapy and/ or anticancer drug therapy, it is suggested to postpone treatment until the complete disappearance of clinical and radiological signs and negativity of COVID-19 (16). However, there are clinical conditions, such as the one here described, that require a prompt oncological treatment due to worsening of symptoms.

To our knowledge, this case describes the first experience of mediastinal radiotherapy in a COVID-19 patient affected by myeloma reported in the literature. The radiation schedule and preventive measures adopted in our case allowed to deliver the radiotherapy treatment safely, without cross contagion of staff and patients. This palliative treatment also allowed self-feeding of the patient, avoiding parenteral nutrition, and improving her quality of life, without worsening of signs and symptoms related to SARS$\mathrm{CoV}-2$ infection.

In terms of study limitations, our experience represents just an isolated case of active cancer treatment delivered to a COVID-19 patient, with a limited follow-up time due to patient's death which, however, was not related to progression of myeloma or SARS-CoV-2 infection.

As the pandemic progresses, it is inevitable that departments will have to deal with decisions to treat or not or to interrupt oncological treatment of SARS-CoV-2 positive patients. In this scenario, sharing insights and experiences, while awaiting further investigations and prospective studies, could improve the global management of oncological treatments and minimize the risk of contagion, while avoiding the undertreatment of patients affected by COVID-19.

\section{Acknowledgments}

Funding: None.

\section{Footnote}

Reporting Checklist: The authors have completed the CARE reporting checklist. Available at http://dx.doi.org/10.21037/ tcr-20-2172

Conflicts of Interest: All authors have completed the ICMJE uniform disclosure form (available at http://dx.doi. org/10.21037/tcr-20-2172). Dr. GG reports personal fees from Abbvie, personal fees from Janssen, personal fees from Astra-Zeneca, personal fees from Sunesys, outside the submitted work. The authors have no other conflicts of interest to declare.

Ethical Statements: The authors are accountable for all aspects of the work in ensuring that questions related to the accuracy or integrity of any part of the work are appropriately investigated and resolved. All the procedures followed were in accordance with the ethical standards of the responsible committee on human experimentation and with the Helsinki Declaration (as revised in 2013). Written 
informed consent was obtained from the patient.

Open Access Statement: This is an Open Access article distributed in accordance with the Creative Commons Attribution-NonCommercial-NoDerivs 4.0 International License (CC BY-NC-ND 4.0), which permits the noncommercial replication and distribution of the article with the strict proviso that no changes or edits are made and the original work is properly cited (including links to both the formal publication through the relevant DOI and the license). See: https://creativecommons.org/licenses/by-nc-nd/4.0/.

\section{References}

1. COVID-19, Cases in Italy 22 May at 6 pm. Available online: http://www.salute.gov.it/portale/nuovocoronavirus/ dettaglioNotizieNuovoCoronavirus.jsp?lingua=italiano\& menu=notizie \&p=dalministero\&id=4799

2. Guan WJ, Ni ZY, Hu Y, et al. Clinical Characteristics of Coronavirus Disease 2019 in China. N Engl J Med 2020;382:1708-20.

3. Bernheim A, Mei X, Huang M, et al. Chest CT Findings in Coronavirus Disease-19 (COVID-19): Relationship to Duration of Infection. Radiology 2020;295:200463.

4. Xia Y, Jin R, Zhao J, et al. Risk of COVID-19 for cancer patients. Lancet Oncol 2020;21:e180.

5. Ueda M, Martins R, Hendrie PC, et al. Managing Cancer Care During the COVID-19 Pandemic: Agility and Collaboration Toward a Common Goal. J Natl Compr Canc Netw 2020. [Epub ahead of print].

6. Krengli M, Ferrara E, Mastroleo F, et al. Running a Radiation Oncology Department at the time of coronavirus: an Italian experience. Adv Radiat Oncol 2020;5:527-30.

7. Filippi AR, Russi E, Magrini SM, et al. Letter from Italy: First practical indications for radiation therapy

Cite this article as: Krengli M, Beldì D, Ferrara E, Zannetti M, Mastroleo F, De Paoli L, Greco M, Matino E, Pirisi M, Gaidano G. Radiotherapy in COVID-19 patient affected by multiple myeloma: a case report. Transl Cancer Res 2020;9(12):7662-7668. doi: 10.21037/tcr-20-2172 departments during COVID-19 outbreak. Int J Radiat Oncol Biol Phys 2020;107:597-9.

8. Guckenberger M, Belka C, Bezjak A, et al. Practice recommendations for lung cancer radiotherapy during the COVID-19 pandemic: An ESTRO-ASTRO consensus statement. Radiother Oncol 2020;146:223-9.

9. Coles CE, Aristei C, Bliss J, et al. International Guidelines on Radiation Therapy for Breast Cancer During the COVID-19 Pandemic. Clin Oncol (R Coll Radiol) 2020;32:279-81.

10. Dai M, Liu D, Liu M, et al. Patients with cancer appear more vulnerable to SARS-COV-2: a multi-center study during the COVID-19 outbreak. Cancer Discov 2020;10:783-91.

11. Mehta V, Goel S, Kabarriti R, et al. Case Fatality Rate of Cancer Patients with COVID-19 in a New York Hospital System. Cancer Discov 2020;10:935-41.

12. Martinez D, Sarria GJ, Wakefield D, et al. COVID's Impact on Radiation Oncology: A Latin American Survey Study. Int J Radiat Oncol Biol Phys 2020;108:374-8.

13. Tsang RW, Campbell BA, Goda JS, et al. Radiation therapy for solitary plasmacytoma and multiple myeloma: guidelines from the International Lymphoma Radiation Oncology Group. Int J Radiat Oncol Biol Phys 2018;101:794-808.

14. Yerramilli D, Xu AJ, Gillespie EF, et al. Palliative Radiotherapy for Oncologic Emergencies in the setting of COVID-19: Approaches to Balancing Risks and Benefits. Adv Radiat Oncol 2020;5:589-94.

15. Rudziakiene M, Inciura A, Gerbutavincius R, et al. Single vs. multiple fractions regimens for palliative radiotherapy treatment of multiple myeloma. Strahlenther Onkol 2017;193:742-9.

16. Raymond E, Thieblemont C, Alran S et al. Impact of the COVID-19 Outbreak on the Management of Patients with Cancer. Target Oncol 2020;15:249-59. 